\title{
Electrical Conductivity of PA6/Graphite and Graphite Nanoplatelets Composites using Two Processing Streams
}

\author{
Umar M., ${ }^{1} *$ Ofem M. I., ${ }^{2}$ Anwar A. S. ${ }^{1}$ and Usman M. M. ${ }^{3}$ \\ ${ }^{1}$ Department of Chemical Engineering, Kaduna Polytechnic, Kaduna, Nigeria \\ ${ }^{2}$ Department of Mechanical Engineering, Cross Rivers University of Technology, Calabar, Nigeria \\ ${ }^{3}$ Department of Chemical Engineering, Federal Polytechnic, Nasarawa, Nigeria \\ Corresponding Author: *michaeliofem@ crutech.edu.ng
}

https://doi.org/10.36263/nijest.2021.01.0251

\begin{abstract}
The percolation threshold (PT) of any polymer/particulate carbon composite depends on the processing, the dispersed state of the filler, the matrix used and the morphology attained. Sonication technique was used to make PA6/G and PA6/GNP composites employing in situ polymerisation, after which their electrical conductivity behaviours were investigated. While overhead stirring and horn sonication were used to distribute and disperse the carbon fillers, the composites were made in 2 streams $40 / 10$ and 20/20. The 40/10 stream implies that while dispersing the carbon fillers in PA6 monomer, $40 \%$ amplitude of sonication was applied for 10 minutes whereas the 20/20 stream implies 20\% amplitude of sonication for 20 minutes. In both streams, the dispersing strain imparted on the monomer/carbon mixture was 400 in magnitude. Purely ohmic electrical conductivity behaviour was attained at $9.75 \mathrm{G} w \mathrm{w}$. \% for IG 40/10 system. For composites in the IG 20/20 system, same was attained at $10.00 \mathrm{G} w t . \%$. Electrical conductivity sufficient for electrostatic discharge applications was achieved above $15 \mathrm{G} w t . \%$ in the IG 40/10 system. Using the power law percolation theory, percolation threshold was attained at $9.7 \mathrm{G} w \mathrm{w} . \%$ loading in IG 40/10 system, while same was attained at $7.6 \mathrm{G}$ wt. \% loading in IG 20/20 system. For the GNP based systems, percolation threshold occurred at 5.2 GNP wt. \% in the INP 40/10 system whereas same occurred at 7.4 GNP wt. \% in the IG 20/20 system.
\end{abstract}

Keywords: Electrical-conductivity, Graphite, Percolation-threshold, Amplitude, Sonication

\subsection{Introduction}

Polymers are essentially insulators except for the natural double conjugates. To introduce electrical conductivity to polymers, conductive constituents such as metals and carbon are used as fillers. Fillers are carefully selected and added in quantity that will not compromise other important physical properties of the matrix. Without much loss to other significant matrix properties, threshold quantities just above which an electrically percolated conductive state is attained is needed. At higher loading above the threshold amount, the risk of the composite becoming too brittle and loss of ability to perform other functions is expected. For the conductive regimes, usefulness is found when the electrical conductivity attained can sufficiently prevent lightning strikes, shield electromagnetic effects, provide anti-statics to components and in applications related to strain sensors (Marsden et al. 2018).

Polymer/micro-particulate composites, such as polymer/G composites, are usually made using high weight percentages of the fillers, sometimes, up to $40 \mathrm{wt}$. \% (Clingerman et al., 2002). This is mainly done to attain significant property improvements such as converting a composite from an electrical insulator to a conductor (Clingerman et al., 2002). This transition takes effect at the percolation threshold (PT) which corresponds to that conductive filler loading level just above which, composites transform from being an electrical insulator to electrical conductor. The transition is usually marked by a sharp rise in electrical conductivity due to the formation of an electrically-conductive network by the filler. A plot of conductivity against filler loading takes the characteristic S-shape which represents the insulating, percolating and conductive regimes (Marsden et al., 2018). 
For each filler/matrix combination, the PT depends on the blending method used, the type, shape, size and orientation of the filler in the polymer composite and the filler-matrix interaction or, how considerable the matrix wets the filler (Clingerman, 2001). Many properties including electrical conductivity of composites may follow the power law percolation theory. When written for electrical conductivity; the critical filler loading at percolation, $p_{c}$, and the power constant, $t$, can be determined from Equation 1 (Marsden et al., 2018);

$\sigma(p)=\sigma_{c}\left(p-p_{c}\right)^{t}$ for $p>p_{c}$

Where $\sigma$, is the specific composite conductivity; $\sigma_{c}$, the conductivity at the filler loading above which the composites conductivity raises in an order of magnitude and, $p$ is the filler loading in wt. \%. The power constant, $t$, indicates the dimensionality of the filler network. Its typical value may range between 1.3-2.0 for systems in which the filler network dimension (D) lays between 2D and 3D (Sandler et al., 2003). As a class of polymer/carbon particulate composites, electrically conductive polymer composites find application in electromagnetic shielding, antistatic protection, batteries (Zheng et al., 2002), in aerospace and sports goods (Krupa and Chodak, 2001).

The use of graphite nanoplatelets (GN) is not often common due to the need of high weight percentage. The GN density of $2.26 \mathrm{~g} / \mathrm{cm}^{3}$ (Sengupta et al., 2011) tends to increase the density of the final products. In addition, the accompanying rise in melt viscosity makes processing more difficult and energy consuming (She et al., 2007). Notwithstanding, using a solution blending process with poly (methyl methacrylate) (PMMA), an electrical conductivity percolation threshold is reported at $3.61 \mathrm{G}$ wt. \% (Moniruzzaman and Winey, 2006). Again, using G, the critical exponent of $t$ (in Equation 1) is approximately 1.8 as reported by Zheng et al. (2002). This suggests that a 3 dimensional conductive filler network was formed. The authors ascribed the low electrical conductivity PT to a 'tunnelling' phenomenon between adjacent conductive clusters. In a comparative study involving $\mathrm{G}$ and expanded graphite (EG) using polystyrene (PS) matrix, PT was attained at $6 \mathrm{G}$ wt.\% (Chen et al., 2003B). In a similar investigation for PMMA/G composites, a PT of 3.19 vol.\% was achieved using in situ polymerisation (Chen et al., 2003A). Both in situ polymerisation and solution blending usually result in lower PT values compared to melt blending (Sengupta et al., 2011). Clingerman (2001) used an additive equation to describe, among other properties, the electrical percolation behaviour of synthetic graphite (SG) here referred to as $\mathrm{G}$ in PA6, 6 and polycarbonate (PC) matrices. The PA6, 6/G composite was estimated to have a PT of about 11 vol. \%. Significant electrical conductivity of $2 \times 10^{-3} \mathrm{~S} / \mathrm{cm}$ was only attained at a much higher loading of $25 \mathrm{vol} \%$. Other studies (She et al., 2007; Lu et al., 2005) of G and EG with polyethylene showed that only at significantly large amounts (22.2 and 18 vol. \% for G and EG, respectively) did composites became electrically conductive.

It is also common for $\mathrm{G}$ composites to show sluggish percolation behaviour. In such instances, the change from insulator to a conductor is not sharp, and the electrical conductivity at PT obtained will not be high enough for many practical applications. For instance, Zheng et al. (2004) melt compounded high density polyethylene, (HDPE) with G; the first steep rise in conductivity occurred at $5 \mathrm{G}$ wt. $\%$ but the conductivity achieved was only $1 \times 10^{-11} \mathrm{~S} / \mathrm{cm}$. However a steeper rise followed at about $8 \mathrm{G}$ wt. $\%$ to give conductivity of about $1 \times 10^{-6} \mathrm{~S} / \mathrm{cm}$ sufficient for electrostatic discharge applications (Moniruzzaman and Winey, 2006).

The high volume fractions of micron-scale fillers such as $\mathrm{G}$ required improving the properties leads to high viscosities during processing. To prevent forming highly dense product, co-blending two or more polymers with the filler helps. The concept being to make the filler preferentially dispersed in one phase while the volume of the other phase becomes effectively excluded (Thongruang et al., 2002). Alternatively, increasing the degree of crystallinity (Gubbel et al., 1995) of composites when a semicrystalline matrix is used can also be used as a strategy. During crystal growth, fillers are pushed aside into the amorphous phase. Mixtures of micron-scale carbon fillers (Clingerman et al., 2002; Elwell et al., 2004) can also be used to attain early electrical conductivity PT.

Adding nano-sized particulate carbon fillers such as GNP can also be made to attain property gains such as electrical conductivity. This could be attained at loading levels where processing the composite is not severely altered compared to the unfilled matrix (Kalaitzidou et al., 2007A). A low 
PT of 0.75 vol. \% was attained using in situ polymerisation of EC to produce PA6/GNP composites (Weng et al., 2004). The highest electrical conductivity attained was $10^{-3} \mathrm{~S} / \mathrm{cm}$. The synthesis was assisted by sonication which continued until the system's viscosity increased. The attainment of low PT was ascribed to good dispersion, the structure and aspect ratio of the GNP. It is observed that washing the product by boiling for one hour will improve conductivity by the elimination of diluents while sonication during synthesis may shorten polymerised chains, reduce molecular weight and increase the levels of water soluble oligomers.

Percolation behaviour of conductive fillers can also be investigated for already conductive conjugated polymers such as Polyaniline (PANI). The electrical conductivity and percolation behaviour of PANI was determined using in situ polymerisation of aniline with GNP where at 0.32 GNP vol. \%, conductivity of $420 \mathrm{~S} / \mathrm{cm}$ was obtained as against $5 \mathrm{~S} / \mathrm{cm}$ for unfilled PANI. The nanocomposites' conductivity increased gradually to $522 \mathrm{~S} / \mathrm{cm}$ at $4.5 \mathrm{vol}$. \% which doubled the former in order of magnitude. Applying percolation theory, a critical exponent of $t=0.85$ was obtained which is lower than the universal value (1.3-2). In this wet process, the impressive result was assigned to the high aspect ratio of GNP which led to the formation of an effective conductive network.

Another wet process, (solution blending) was employed by Vadukumpully et al. (2011) in an effort to determine the PT in poly (vinyl chloride)/GNP nanocomposites. Relative to the unfilled PVC for composites with GNP loading level less than 0.1 vol. \%, no electrical conductivity was attained. However, some electrical conductivity was attained from 0.1-0.6 GNP vol. \% which was not even sufficient to attain the basic practical applications such as preventing the accumulation antistatic charges. Useful electrical conductivity was attained from $1.3 \mathrm{GNP}$ vol. \%. The authors reported that the rapid rise in electrical conductivity around $6 \mathrm{GNP}$ vol. \% was assumed to correspond with the PT. Nonetheless, wet processing does not always guarantee low PT for polymer/GNP composites although it is always likely to be lower relative to melt processing. In a comparative study, high PT values were obtained at 12 and 15 wt. \% for solution blending and counter-rotating twin-screw extrusion respectively using low linear density polyethylene (LLDPE)/GNP nanocomposites. The GNP loading levels were up to $20 \mathrm{wt}$ \% (Kim et al., 2009). Here, the solution process was employed alongside a series of twin-screw extrusion melt compounding processes. Overall, solution mixing gave the best dispersion. Among the melt compounded composites; the counter-rotating screw arrangement gave the highest PT. Reasons for the high PT values were not given, however, the GNP aspect ratio of 1500 suggests that it was GNP-15 which has a moderate surface area of $100 \mathrm{~m}^{2} / \mathrm{g}$ (Kalaitzidou et al., 2007C). DSC results showed decreased crystallinity relative to unfilled LLDPE on both the solution blending and melt compounding. This decrease does not favour the lowering of PT (Zang et al., 2007).

Kalaizidou et al. (2007C) made a series of investigations on the properties PP/GNP nanocomposites. The objective is to study the effect on the electrical conductivity of three mixing methods namely; melt compounding, solvent processing and polymer coating/melt compounding. The processes are followed by compression or injection moulding. Two GNP fillers, GNP-1 and GNP-15 with the same surface area of about $100 \mathrm{~m}^{2} / \mathrm{g}$ were used with the latter having higher aspect ratio. Polymer coating followed by melt compounding was found to be as efficient as solvent processing. SEM micrographs indicated uniform dispersion of the GNP particles in the polymer coated/melt compounded nanocomposites. The authors observed that at the same volume fraction, GNP-1 had about $200 \mathrm{x}$ more particles than GNP-15. This increased the ability of GNP-1 to efficiently coat PP there by inducing electrical conductivity at lower loadings. With regard to the GNP-coated melt compounded nanocomposites (the best among the melt processed systems), followed with compression moulding gave better properties than injection moulding. The complex 3D particle orienting effects found in the fountain-flow in injection moulding as against the 2D planar orientation generated during compression moulding may have contributed to this (Kim and Mascoko, 2009). For the coated melt compounded/injection moulded nanocomposites of GNP-15, PT was attained at 5 vol. \%. When uncoated melt extrudates with injection moulded, PT was attained at approximately 7 vol. $\%$.

In a related investigation by Kalaizidou et al. (2007B), an SEM micrograph showed that due to the higher surface area of GNP-15 (implying more flexibility) it may fold and roll up. Therefore, it suffices to say that the PT of any polymer/particulate carbon composite depends on the processing, the dispersed state of the filler, the matrix used and the morphology attained. Fukushima and Drzal 
(2006) conducted a research to identify the PT of PA6, 6/carbon nanocomposites using co-rotating twin screw extrusion, the carbon fillers used were GNP-1 and GNP-15. PA6, 6/GNP-15 had a lower PT of 6 vol. \% compared to the PT of PA6, 6/GNP-1 at 10-12 vol. \%. The variation could be attributed to the processing which allowed the retention of the natural morphology of GNP-15 leading to early formation of an electrically-conductive percolated network. Just as high aspect ratio is expected to decrease thermal contact resistance (Kalaitzidou et al., 2007C) so it is expected to facilitate smooth flow of electric charges.

In the light of the above in this research, a micro-composite PA6/graphite (G) and a nanocomposite, PA6/graphite nanoplatelets (GNP) are synthesized in situ employing a novel approach. Overhead stirring and horn sonication will be used to distribute and disperse the carbon fillers, using two streams 40/10 and 20/20. The 40/10 stream implies that while dispersing the carbon fillers in PA6 monomer, $40 \%$ amplitude of sonication will be applied for 10 minutes while the 20/20 stream implies that $20 \%$ amplitude of sonication was applied for 20 minutes. The electrical conductivity for the two streams at different filler loading will be investigated. At what filler loading will 'purely ohmic behaviour' of composite be achieved? The percolation threshold (PT) for the two streams will be examined for the in situ for graphite (IG) and in situ polymerised for nanoplatelets (INP).

\subsection{Methodology}

\subsection{Materials}

Pristine commercial grade PA6 was donated by Akulun Germany. The monomer Epsilon Caprolactam (C6H11NO, coded as EC) was purchased from Sigma-Aldrich, with purity level of $99 \%$ and a molecular weight of 113.16. The pristine PA6 and the EC were vacuum dried overnight at $50^{\circ} \mathrm{C}$ before usage which adequately removed moisture. Methyl Magnesium Bromide (coded as MMB, molecular weight 119.26,) is a Grignard catalyst precursor which forms the catalyst, (Caprolactam Magnesium Bromide (CMB)) in-situ, was purchased Fisher Scientific as a $100 \mathrm{ml}$ bottle containing a 3.0 $\mathrm{M}$ solution of $\mathrm{MMB}$ in diethyl ether. Activator: or co-catalyst, is a monofunctional Nacetylcaprolactam $\left(\mathrm{C}_{8} \mathrm{H}_{13} \mathrm{NO}_{2}\right)$, coded as NAC), supplied by Sigma-Aldrich with purity; $99 \%$ and molecular weight of 155.19. Graphite Filler: Synthetic graphite, (coded as G) is $\leq 2 \mu \mathrm{m}$ in particle size was supplied by Sigma-Aldrich. Graphite Nano-Platelets: GNP-15, (coded as GNP) with surface area $107 \pm 7 \mathrm{~m}^{2} / \mathrm{g}$, diameter of $15 \mu \mathrm{m}$, aspect ratio of 1500 and density of $2 \mathrm{~g} / \mathrm{cm} 3$ was bought from XGSciences, UK. Prior to use G and GNP are kept overnight in an oven at $160{ }^{\circ} \mathrm{C}$.

\subsection{Methods}

Details of production of composite can be found elsewhere (Umar et al. 2020). Briefly, graphite (G) and graphite nanoplatelets (GNP) were cumulatively added to the molten monomer EC and dispersed by simultaneous sonication and mechanical stirring. This was followed by the addition of the catalysing species at the designated temperatures. The reaction took place in situ in a $100 \mathrm{ml}$ beaker which served as the reaction vessel. Catalyst precursor and a co-catalyst were employed to ensure that the reaction to form the composites occurred. For the PA6/G composites, $\mathrm{G}$ loading ranged between 5-25G wt. \% loading while for the GNP, the loading was between 0.5-2.5 GNP wt. \%. Two similar sonication processing streams with magnitudes theoretically similar in processing streams were used. In one, the amplitude of sonication was kept at $40 \%$ and sonication was conducted for $10 \mathrm{mins}$. In the other, sonication was conducted for double the time, 20mins but half the sonication amplitude, $20 \%$. The first was designated as the IG 40/10 and IGNP 40/10 streams while the others are the IG20/20 and IGNP 20/20 streams. After the synthesis solid products were withdrawn from the reaction vessel and crushed without washing while, dog bone specimens were prepared on a Haake Mini-Lab injection moulding machine. To prepare the specimens for electrical conductivity the straight and central portions of the dog-bones were used for all the carbon loadings and for unfilled PA6.

\subsection{Characterization for eectrical conductivity}

The electrical conductivity of pure PA6, G- and GNP-based composites were investigated using an impedance spectrometer; a numeric Q phase sensitive multi-meter (PSM 1735). The meter is a 2 channelled, 4 wire kind which was operated in the inductance, current and resistance or LCR mode. The resistance displayed by test specimen as the frequency of the applied voltage varied from 1-10 $\mathrm{Hz}$ indicates the electrical conductivity of the measured test specimen. 


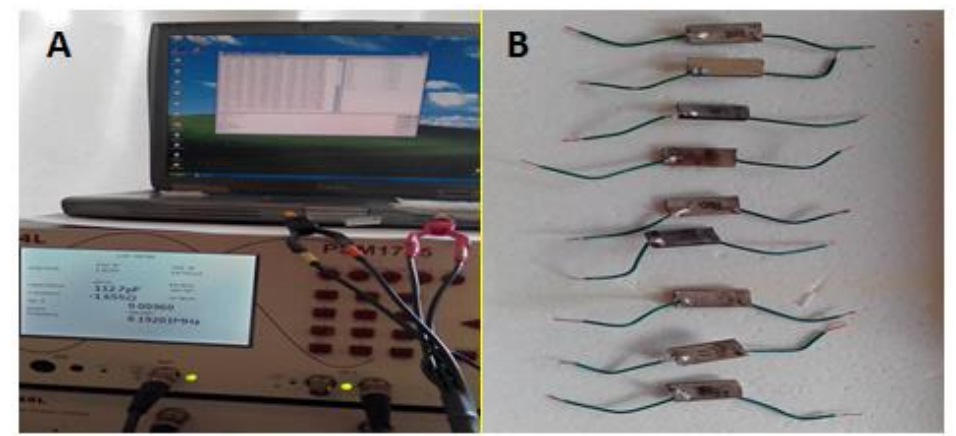

Figure 1: (A) Numeric Q phase sensitive multi-meter (PSM 1735) used to measure electrical conductivity, (B) specimens prepared for testing

A specimen's electrical conductivity was measured across its thickness and perpendicular to the direction of any filler alignment imposed by injection moulding. Silver paint was applied on both faces and specimens were then allowed to dry. Using a silver-epoxy conductive paste, copper wires were alternately attached on opposite faces. Figure 1A and 1B shows the test set up and ready-to-test specimens. Each specimen test was repeated 3 times. For all the carbon loadings at least 5 separate specimens were tested. The resistance recorded was converted to electrical conductivity in S/m using the dimensions of the samples according to Equation 2.

$\sigma(\mathrm{S} / \mathrm{m})=\frac{1}{\mathrm{R}} \mathrm{X} \frac{\text { Specimen thickness }}{\text { specimens'cross sectional area }}$

where $\sigma$ is the electrical conductivity; $R$ is the resistance of the materials.

\subsection{Results and Discussions}

\subsection{Electrical conductivity behaviour for in situ polymerised PA6/G composites (IG 40/10 and the IG 20/20 Systems)}

Figures 2A and 2B depicts frequency dependence conductive behaviour of 2 in situ polymerised PA6/GNP composites systems, IG 20/20 and IG 40/10. In both, the conductivity patterns remain somewhat similar even as the frequency of the current increases at higher carbon loadings. The frequency dependence of electrical conductivity for both systems show a 'purely ohmic behaviour' (Sandlera et al., 1999) as can be seen in the figures. This was achieved at $9.75 \mathrm{G}$ wt. \% loading in IG $40 / 10$ system and above $10 \mathrm{G}$ wt. \% loading in IG 20/20 system. The relatively early lower frequency independent electrical conductivity in IG 40/10 system may be related to the higher tendency of its broken $\mathrm{G}$ particles agglomerate to form a conductive path. The sonication amplitude of $40 \%$ breaks more particles reducing their aspect ratios. The smaller the particles are, the more their tendency to attract each other and agglomerate. Agglomeration supports the formation of an electrically conductive network (Alig et al., 2008). In some cases, lower aspect ratio particles cause the formation of an electrically conductive path at lower carbon content. This is due to higher number of particles at equivalent weight fractions in comparison to higher aspect ratio particles (Kalaitzidou et al., 2007A). Such promotes the formation of a conductive path via agglomeration. On the one hand, with the smaller sized particle, surface tension increases in polymers (Supova et al., 2011) while particle/particle attraction increases making it easier formation of agglomeration-based conductive path. Again, the tendency for particles to agglomerate increases with decreasing particle size (Keledi et al., 2012) though with smaller sized particles, transport resistances also increases at particle/particle junctions. 

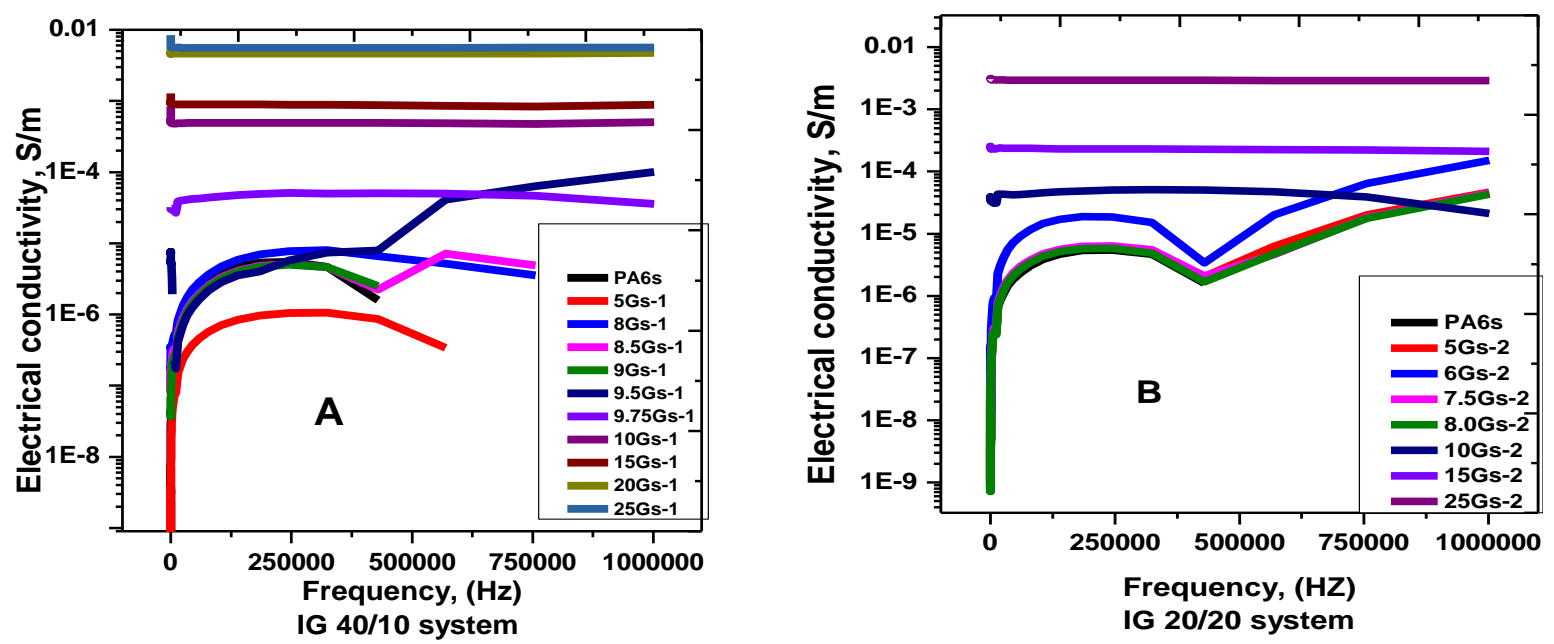

Figure 2: Frequency dependence of electrical conductivity in-(A) IG 40/10 system (sonication amplitude of $40 \%$ applied for 10 minutes) (B) IG 20/20 system (20\% amplitude applied for $20 \mathrm{~min}$ )

Figure 3 depicts the percolation behaviour of the IG 40/10 system by fitting it to the power law equation. It seemed not to correspond well with the commonly reported sigmoidal curve trends. A similar behaviour with carbon loading, the electrical conductivity continues to grow defying the sigmoidal curve pattern was previously reported (Chandrasekaran et al., 2013). Such trends may suggest that there is a significant growth in the volume of the conductive path formed above critical carbon loading. In the IG 40/10 system, electrical conductivity sufficient for electrostatic discharge applications $\left(10^{-5}-10^{-4} \mathrm{~S} / \mathrm{m}\right.$ (Moniruzzaman and Winey, 2006)) is achieved above $15 \mathrm{G}$ wt \%. Kang and Chung (2003) attained this by incorporating 8 wt. \% G and 4 wt. \% oil in a PA6 hydrolytic based in situ polymerisation process. Most likely, oil had excluded a significant volume of the composite thereby reducing the volume required to form a conductive path.

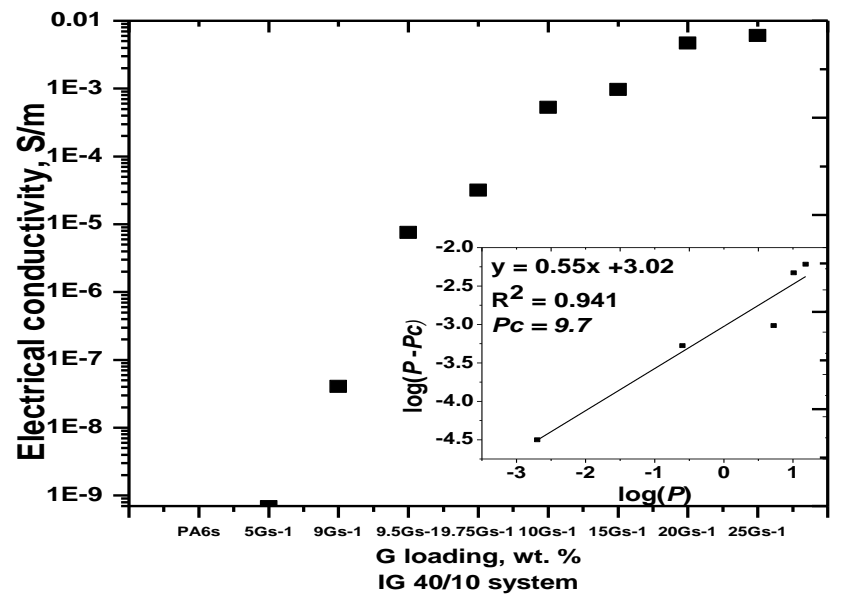

Figure 3: Electrical conductivity percolation curve for the G 40/10 system (40\% sonication applied for $10 \mathrm{~min})$. Inset is the corresponding power law fit

The inset in Figure 3 is the power law fitting of the percolation curve. The estimated percolation threshold (PT) loading is about $9.7 \mathrm{G}$ wt. \% which is significantly lower than some reported values in studies containing polymer/G composites (Kang and Chung, 2003; Chen et al., 2003B; Chen et al., 2001). This observed disparity may have to do with the variation in the matrix type, the effect arising from same and the inclusion of oil. Crystal formation in semi-crystalline polymers like PA6 also enhances electrical conductivity. As crystals are formed they push away filler particles thereby narrowing the total volume required to form a conductive path (Krupa and Chodák, 2001). When a very low PT loading of close to $6 \mathrm{G}$ wt. \% was reported with a HDPE matrix (Zheng et al., 2004), the success was associated with filler shape, the mixing viscosity and the effect of the actual blending process (Cheng et al., 2010). Additionally all polymers differ in their intrinsic dielectric properties. Although in situ polymerisation (as applied here ) has the advantage of providing good wetting of carbon fillers (Chen et al., 2003A), it also limits direct conduction of electric current since the carbon particles become coated (Weng et al., 2005). A similar effect also occurs when the filler is 
encapsulated, for instance when master-batch processing precedes melt extrusion. In such cases, electron flow switches from a contact-based mechanism into an electron tunnelling type (Li and Chen, 2007).

In percolation data fittings, the critical constant, $t$, describes the shape of the fillers as it relates to the dimensionality of the conductive path attained (Weng et al., 2005; Yamamuro et al., 1999). In the G $40 / 10$ system, a critical constant of 0.55 is obtained with the regression coefficient above 0.9 which justifies the value. Conventionally, $t$ values close to 2 implies that the electrical conductive network is 3 dimensional (Schmidt et al., 2007). Deviations to higher values are considered to be a manifestation of a non-universal phenomenon (Foulger, 1999B; Chen et al., 2003A; Foulger, 1999A). Chen et al. (2003A) suggests $t$ values between 1.65 to 2.0 to correspond to a 3 dimensional filler connectivity network while, extreme filler geometry causes deviations to higher values. This results in other forms of conductive mechanism, for instance tunnelling (Foulger, 1999B) to occur in parallel with the contact type. Filler connectivity can also be such that a two dimensional network forms. Values around 1.1 (Yamamuro et al., 1999), those within the range of 1.2-1.30 (Schmidt et al., 2007) and even up to 1.33 (Bauhofer and Kovacs, 2009) have all been considered to describe an electrically conductive path with a dimensionality of 2 . Values of $t$ far away from the range typically associated with both the two and three dimensional connectivity are sometimes observed especially when, sonication is used in dispersing the fillers (Bauhofer and Kovacs, 2009). For instance, a $t$ value of 7.6 (Ha et al., 2007) was obtained as well as a $t$ value of less than 1 (Wang and Dang, 2005), both in processes involving carbon dispersion by sonication. Therefore, the $t$ value of $\approx 0.6$ obtained in the $\mathrm{G}$ $40 / 10$ system is not an isolated finding. It appears that the harsh sonication conditions applied in $40 / 10$ created small particles of diverging geometries as evident in Figure 4 below. The red arrow points at somewhat distorted cone among other intricate shapes. Such mixed geometries are anisotropic thus, lacking any definable central or rotational symmetry to fit proper aspect ratio description (Supova et al., 2011) .

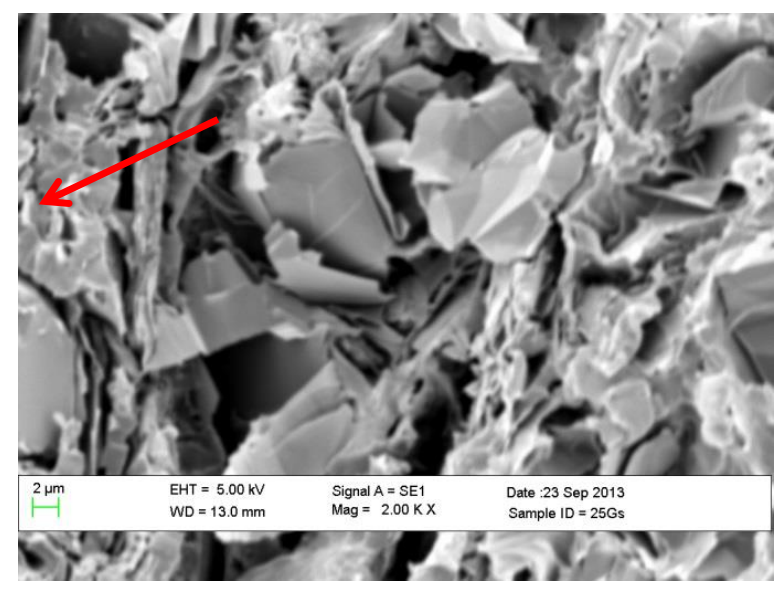

Figure 4: SEM photo for tensile fractured PA6/G of the IG 40/10 system. (40\% sonication applied for $10 \mathrm{~min}$ )

Figure 5 shows the PT fitting for IG 20/20 system where the PT forms at a lower loading of $7.6 \mathrm{G}$ wt. $\%$ with a regression coefficient, $\mathrm{R}=0.994$. The PT value compares favourably with that achieved in $\mathrm{G} 40 / 10$ system at $9.7 \mathrm{G}$ wt. \% with $\mathrm{R}=0.941$. This is an indication of a better retention of the aspect ratio in G 20/20 system despite a higher value of the sonication. The $t$ value of 2.26 obtained here is an approximate to a 3 dimensional connectivity network as reported in literature (Bryning et al., 2005; Yuen et al., 2007; Yoshino et al., 1999; Mierczynska et al., 2007). It also shows that formation of an electrically conductive network occurred preferentially with a well dispersed $\mathrm{G}$ compared to that based on agglomerated conductive path. 


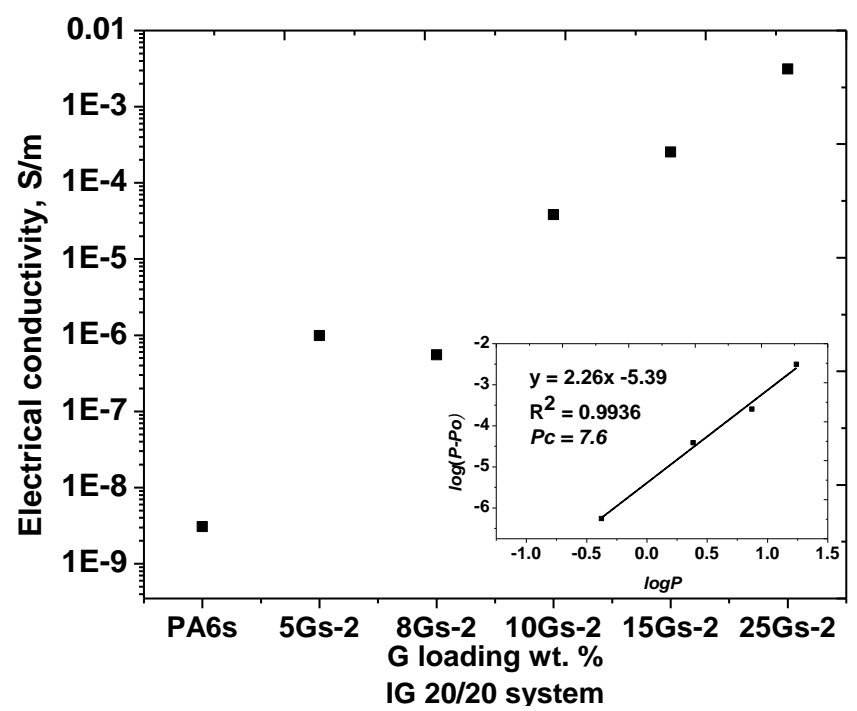

Figure 5: Electrical conductivity percolation curve for IG 20/20 system where 20\% amplitude of sonication was applied for $20 \mathrm{~min}$. Inset is the corresponding power law fit

3.2. Electrical conductivity behaviour for in situ polymerised PA6/G composites (INP 40/10 and the INP 20/20 systems)

A significant deviation of $t$ values found in literature occurs in the INP 40/10 system as represented in Figure 6. The deviation is believed to be strongly associated with the nano filler size and shape of the GNP due to 40/10 harsh sonication conditions. Formation of large quantity of relatively small particles of different shapes is linked to sonication conditions. There seems to be no studies with which to compare these results, i.e. where GNP is incorporated in situ during synthesis of PA6 using fast anionic polymerisation which follows concurrent direct horn sonication and mechanical stirring. The two dimensional lateral structure of GNP combined with harsh sonication conditions can alter the GNP geometry and as such, the measured $t$ value of 22.7 may not be unusual since a value close to 8 was reported when one dimensional CNT was dispersed by sonication (Ha et al., 2007). Ideally, $t$ values describe both the geometry and dimensionality of connectivity networks (Foulger, 1999A; Foulger, 1999B). The percolation theory may have limitations when it comes to carbon particles as reported by Gojny et al. (2006) where higher PT values are predicted. The higher PT for INP 40/10 system is also likely associated to filler agglomeration on the nano-scale. This can negate the formation of a sufficiently large conductive path all across the specimen. The degree of crystallisation (DoC) reported in Marsden et al. (2018) did not vary significantly, relatively higher DoC did occur for INP 40/10 system. The lowest and highest values are 33.9 \pm 0.9 and $37.8 \pm 1.0 \mathrm{GNP}$ wt. \% respectively. This compares to $26.0 \pm 0.9$ and $35.7 \pm 1.7 \mathrm{GNP}$ wt. \% in the INP $20 / 20$ system. Hence INP 40/10 which has a higher DoC is more likely to have a higher volume excluded. This will surely aid formation of a lower PT in INP 40/10 system. 


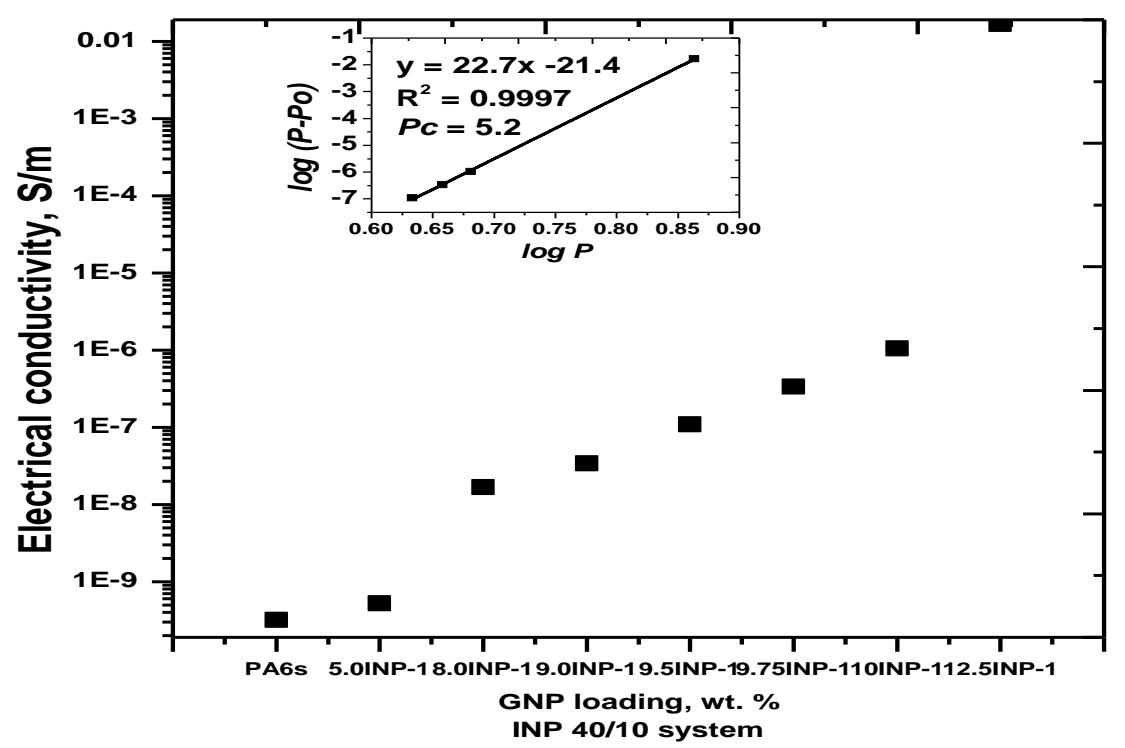

Figure 6: Electrical conductivity percolation curve for the INP 40/10 system where $40 \%$ sonication amplitude applied for $10 \mathrm{~min}$. Inset is the corresponding power law fit

In the INP 20/20 system, electrical conductivity improvement seems quiet gradual as depicted in Figure 7. The effect of a milder sonication manifests with $t$ value of 7.4 which remains within reported range where sonication is applied (Ha et al., 2007). It also agrees with the micrographs shown in Figure 8 where, better maintenance of the lateral structure of the GNP occurs in A for the INP 20/20 with a milder sonication condition. Under the 40/10 sonication condition, the GNP has a more fragmented structure. The higher PT value of 7.4 in the INP 20/20 system relative to 5.2 in the INP 40/10 system gives a difference of 2.2 GNP wt. \% and most likely corresponds to their percolation behaviours.

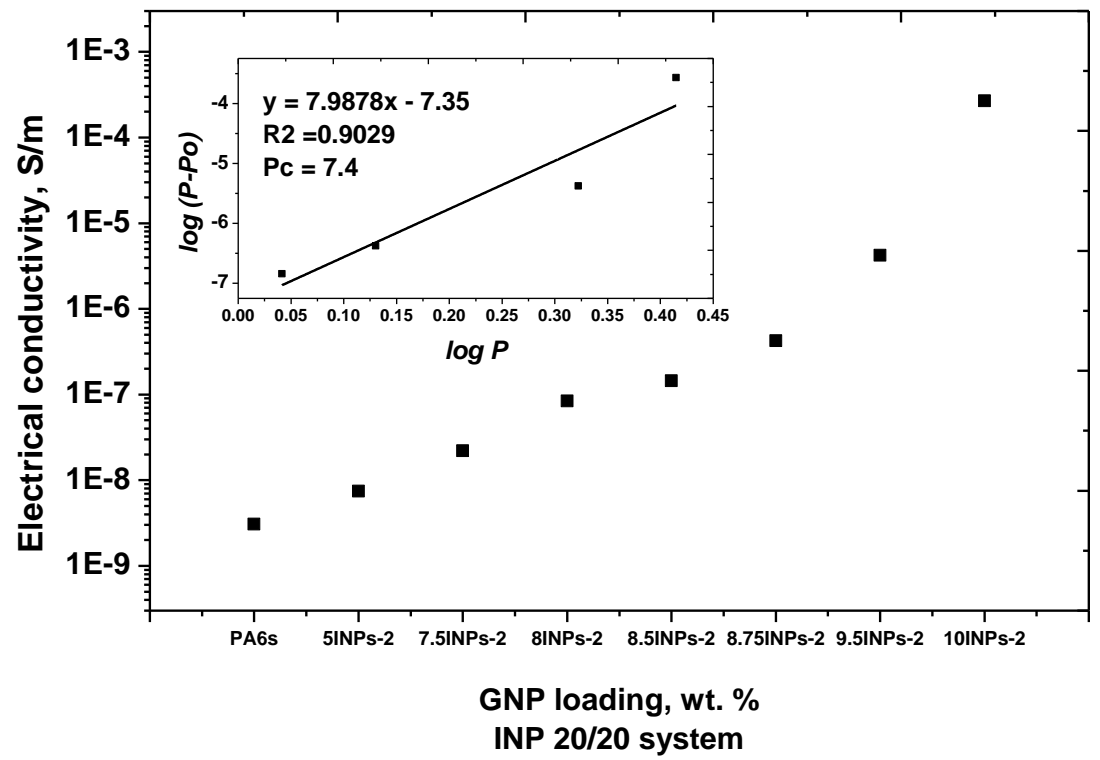

Figure 7: Percolation curve for the INP 20/20 system where 20\% sonication amplitude was applied for $20 \mathrm{~min}$. Inset is the corresponding power law fitting 
A

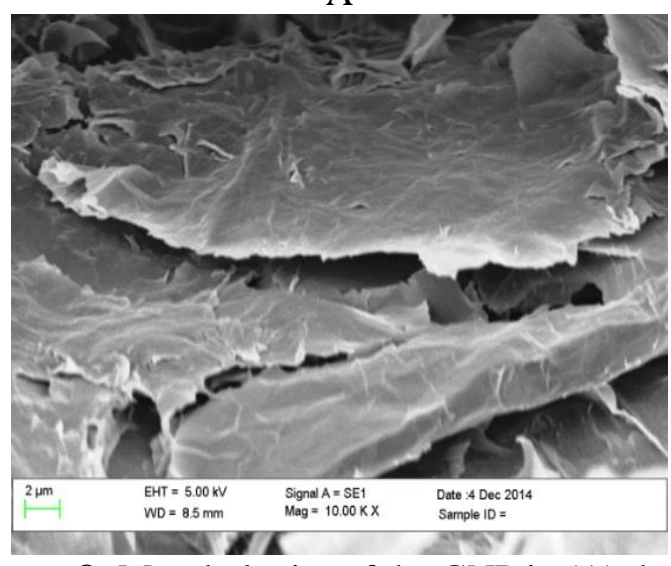

$\mathrm{B}$

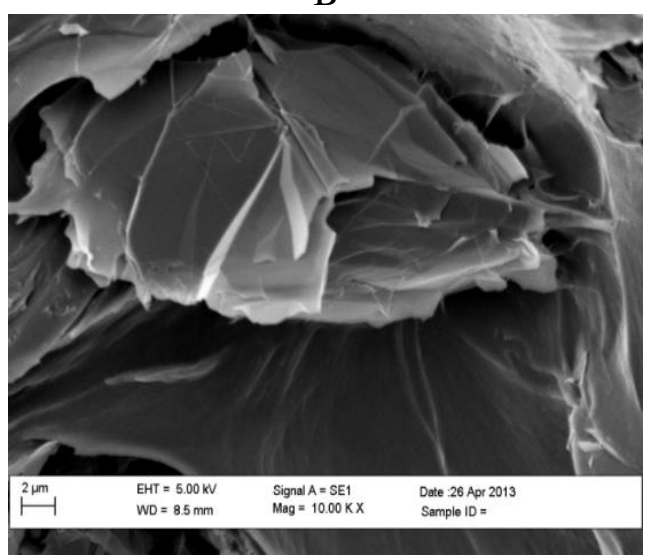

Figure 8: Morphologies of the GNP in (A) the INP 20/20 system (sonication amplitude of $20 \%$ for 20 $\mathrm{min}$ ), and (B) the INP 40/10 system (40\% amplitude for $10 \mathrm{~min}$ )

\subsection{Conclusion}

Using in situ polymerisation and two processing streams of theoretically equivalent carbon filler dispersion strain rates, micron and nano sized carbon composites of PA6 were made and their electrical conductivities and percolation thresholds were investigated. The two dispersion processing streams used were the $40 \%$ amplitude for $10 \mathrm{~min}(40 / 10)$ and the sonication amplitude of $20 \%$ for 20 min (20/20). Each G and GNP composites were made at five cumulative filler loading levels. A purely ohmic electrical conductivity behaviour was attained at $9.75 \mathrm{G}$ wt. $\%$ for the $\mathrm{G}$ composites in IG 40/10 system. For composites in the IG 20/20 system, ohmic electrical conductivity was attained at $10.00 \mathrm{G}$ wt. \%. Percolation threshold was attained at $9.7 \mathrm{G}$ wt. \% loading in IG 40/10 system while, same was attained at $7.6 \mathrm{G}$ wt. \% loading in IG 20/20 system. For the GNP based systems, percolation threshold occurred at 5.2 GNP wt. \% in the INP 40/10 system whereas same occurred at 7.4 GNP wt.\% in the IG 20/20 system.

\section{References}

Alig, I., Skipa, T., Lellinger, D. and Pötschke, P. (2008). Destruction and formation of a carbon nanotube network in polymer melts: rheology and conductivity spectroscopy. Polymer, 49(16), pp. 3524-3532.

Bauhofer, W. and Kovacs, J. Z. (2009). A review and analysis of electrical percolation in carbon nanotube polymer composites. Composites Science and Technology, 69(10), pp. 1486-1498.

Bryning, M. B., Islam, M. F., Kikkawa, J. M. and Yodh, A. G. (2005). Very Low Conductivity Threshold in Bulk Isotropic Single-Walled Carbon Nanotube-Epoxy Composites. Advanced Materials, 17(9), pp. 1186-1191.

Chandrasekaran, S., Seidel, C. and Schulte, K. (2013). Preparation and characterization of graphite nano-platelet (GNP)/epoxy nano-composite: Mechanical, electrical and thermal properties. European polymer journal, 49(12), pp. 3878-3888.

Chen, G., Weng, W., Wu, D. and Wu, C. (2003A). PMMA/graphite nanosheets composite and its conducting properties. European Polymer Journal, 39(12), pp. 2329-2335.

Chen, G., Wu, C., Weng, W., Wu, D. and Yan, W. (2003B). Preparation of polystyrene/graphite nanosheet composite. Polymer, 44(6), pp. 1781-1784.

Chen, G. H., Wu, D. J., Weng, W. G., He, B. and Yan, W. (2001). Preparation of polystyrenegraphite conducting nanocomposites via intercalation polymerization. Polymer international, 2001. 50(9), pp. 980-985. 
Cheng, H. K. F., Sahoo, N. G., Pan, Y., Li, L., Chan, S. H., Zhao, J. and Che, G. (2010). Complementary effects of multiwalled carbon nanotubes and conductive carbon black on polyamide 6. Journal of Polymer Science Part B: Polymer Physics, 48(11), pp. 1203-1212.

Clingerman, M. L. (2001). Development and modelling of electrically conductive composite materials, PhD Thesis Michigan Technological University England.

Clingerman, M. L., Weber, E. H., King, J. A. and Schulz, K. H. (2002). Synergistic effect of carbon fillers in electrically conductive nylon 6, 6 and polycarbonate based resins. Polymer Composites, 23(5), pp. 911-924.

Elwell, J. King, J., Konell, J. and Sutter, L., (2004). Shielding effectiveness of carbon-filled nylon 6,6. Polymer Composites, 25, pp. 407- 416.

Foulger, S. H. (1999A). Reduced percolation thresholds of immiscible conductive blends. Journal of Polymer Science Part B Polymer Physics, 37(15), pp.1899-1910.

Foulger, S. H. (1999B). Electrical properties of composites in the vicinity of the percolation threshold. Journal of Applied Polymer Science, 72(12), pp.1573-1582.

Fukushima, H. and Drzal. L. T., (2006). Nylon-exfoliated graphite nanoplatelet (xGnP) nanocomposites with enhanced mechanical, electrical and thermal properties. Technical Proceedings of the 2006 NSTI Nanotechnology Conference and Trade Show, (NSTI-Nanotech 2006), 1, pp. 282285.

Gojny, F. H., Wichmann, M. H. G., Fiedler, B., Kinloch, I., Bauhofer, W., Windle, A. and Karl, S. (2006). Evaluation and identification of electrical and thermal conduction mechanisms in carbon nanotube/epoxy composites. Polymer, 47(6), pp. 2036-2045.

Gubbels, F., Blacher, S., Vanlathem, E., Jerome, R., Deltour, R., Brouers, F. and Teyssie, Ph (1995). Design of electrical composites: determining the role of the morphology on the electrical properties of carbon black filled polymer blends. Macromolecules, 28(5), pp. 1559-1566.

Ha, M., Grady, B., Lolli, G., Resasco, D. and Ford, W. (2007). Composites of Single-Walled Carbon Nanotubes and Styrene-Isoprene Copolymer Latices. Macromolecular chemistry and physics, 208(5), pp. 446-456.

Keledi, G., Hári, J. and Pukánszky, B. (2012). Polymer nanocomposites: structure, interaction, and functionality. Nanoscale, 4(6), pp. 1919-1938.

Kalaitzidou, K., Fukushima, H. and Drzal, L. T. (2007A). A new compounding method for exfoliated graphite-polypropylene nanocomposites with enhanced flexural properties and lower percolation threshold. Composites Science and Technology, 67(10), pp. 2045-2051.

Kalaitzidou, K., Fukushima, H. and Drzal, L. T. (2007B). Mechanical properties and morphological characterization of exfoliated graphite-polypropylene nanocomposites. Composites Part A: Applied Science and Manufacturing, 38(7), pp. 1675-1682.

Kalaitzidou, K., Fukushima, H. and Drzal, L.T. (2007C). Multifunctional polypropylene composites produced by incorporation of exfoliated graphite nanoplatelets. Carbon, 45(7), pp. 1446-1452.

Kang, S.-c. and Chung, D.-w. (2003). Improvement of frictional properties and abrasive wear resistance of nylon/graphite composite by oil impregnation. Wear, 254(1), pp.103-110.

Kim, H. and Macosko, C. W. (2009). Processing-property relationships of polycarbonate/graphene composites. Polymer, 50(15), pp. 3797-3809. 
Krupa, I. and Chodák, I. (2011). Physical properties of thermoplastic/graphite composites. European Polymer Journal, 37(11), pp. 2159-2168.

Li, J., Sham, M. L., Kim, J. and Marom, G. (2007). Morphology and properties of UV/ozone treated graphite nanoplatelet/epoxy nanocomposites. Composites Science and Technology, 67(2), pp. 296305 .

Lu, J. R., Weng, W. G., Chen, X. F., Wu, D. J., Wu, C. L. and Chen, G. H. (2005). Piezoresistive Materials from Directed Shear-Induced Assembly of Graphite Nanosheets in Polyethylene. Advanced functional materials, 15(8), pp. 1358-1363.

Marsden, A. J., Papageorgiou, D. G., Vallés, C., Liscio, A. Palermo, V., Bissett, M. A., Young, R. J. and Kinloch, I. A. (2018). Electrical percolation in graphene-polymer composites. 2D Materials 5(3), pp. 2003-2021.

Moniruzzaman, M. and Winey, K. I. (2006). Polymer Nanocomposites Containing Carbon Nanotubes. Macromolecules, 39(16), pp. 5194-5205.

Mierczynska-Vasilev, A., Mayne-L'Hermite, M., Boiteux, G., Jeszka, J. (2007). Electrical and mechanical properties of carbon nanotube/ultrahigh-molecular-weight polyethylene composites prepared by a filler prelocalization method. Journal of Applied Polymer Science, 105(1), pp. 158-168.

Sandler, J., Kirk, J. E., Kinloch, I., Shaffer, M. S. P. and Windle, A. (2003). Ultra-low Electrical Percolation Threshold in Carbon-Nanotube-Epoxy Composites. Polymer, 44(19), pp. 5893-5899.

Sandlera, J., Shaffera, M. S. P., Prasseb, T., Bauhoferb, W., Schultea, K. and Windle, A. H. (1999). Development of a dispersion process for carbon nanotubes in an epoxy matrix and the resulting electrical properties. Polymer, 40(21), pp. 5967-5971.

Schmidt, R., Kinloch, I., Burgess, A. and Windle, A. (2007). The Effect of Aggregation on the Electrical Conductivity of Spin-Coated Polymer/Carbon Nanotube Composite Films. Langmuir: the ACS journal of surfaces and colloids. 23(10), pp. 5707-5712.

Sengupta, R., Bhattacharya, M., Bandyopadhyay, S. and Bhowmick, A. K. (2011). A review on the mechanical and electrical properties of graphite and modified graphite reinforced polymer composites. Progress in Polymer Science, 36(5), pp. 638-670.

She, Y., Chen, G. and $\mathrm{Wu}, \mathrm{D}$. (2007). Fabrication of polyethylene/graphite nanocomposite from modified expanded graphite. Polymer International, 56(5), pp. 679-685.

Supova, M., Martynkova, G. S. and Barabaszova, K. (2011). Effect of nanofillers dispersion in polymer matrices: a review. Science of Advanced Materials, 3(1), pp. 1-25.

Thongruang, W., Balik, C. M., and Spontak, R. J. (2002). Volume-exclusion effects in polyethylene blends filled with carbon black, graphite, or carbon fiber. Journal of Polymer Science Part B: Polymer Physics, 40(10), pp. 1013-1025.

Umar, M., Ofem, M. I. Anwar, A. S. and Salisu, A. G. (2020). Thermo gravimetric analysis (TGA) of PA6/G and PA6/GNP composites using two processing streams. Journal of King Saud University Engineering Sciences (in the press) doi.org/10.1016/j.jksues.2020.09.003

Vadukumpully, S., Paul, J., Mahanta, N. and Valiyaveettil, S. (2011). Flexible conductive graphene/poly (vinyl chloride) composite thin films with high mechanical strength and thermal stability. Carbon, 49(1), pp. 198-205.

Wang, L. and Dang, Z.-M. (2005). Carbon nanotube composites with high dielectric constant at low percolation threshold. Applied Physics Letters, 87(4), pp. 042903-042905. 
Weng, W., Chen, G., Wu, D., Chen, X., Lu, J. and Wang, P. (2004). Fabrication and characterization of nylon 6/foliated graphite electrically conducting nanocomposite. Journal of Polymer Science Part B: Polymer Physics, 42(15), pp. 2844-2856.

Weng, W., Chen, G. and Wu, D. (2005). Transport properties of electrically conducting nylon 6/foliated graphite nanocomposites. Polymer, 46(16), pp. 6250-6257.

Yamamuro, S., Sumiyama, K., Hihara, T. and Suzuki, K. (1999). Geometrical and electrical percolation in nanometre-sized Co-cluster assemblies. Journal of Physics: Condensed Matter, 11(16), pp. 3247

Yoshino, K., Kajii, H., Araki, H., Sonoda, V., Take, H. and Lee, S. (1999). Electrical and optical properties of conducting polymer-fullerene and conducting polymer-carbon nanotube composites. Fullerene science and technology, 7(4), pp. 695-711.

Yuen, S., Ma, C., Wu, H., Kuan, H., Chen, W., Liao, S., Hsu, C. and Wu, H. (2007). Preparation and thermal, electrical, and morphological properties of multiwalled carbon nanotube and epoxy composites. Journal of Applied Polymer Science, 103(2), pp. 1272-1278.

Zhang, W., Dehghani-Sanij, A. A. and Blackburn, R. S. (2007). Carbon based conductive polymer composites. Journal of materials science, 42(10), pp. 3408-3418.

Zheng, W., Wong, S.-C. and Sue, H. J. (2002). Transport behavior of PMMA/expanded graphite nanocomposites. Polymer, 43(25), pp. 6767-6773.

Zheng, W., Lu, X. and Wong, S. C. (2004). Electrical and mechanical properties of expanded graphite-reinforced high-density polyethylene. Journal of Applied Polymer Science, 91(5), pp. 27812788.

Cite this article as:

Umar M., Ofem M. I., Anwar A. S. and Usman M. U., 2021. Electrical Conductivity of PA6/Graphite and Graphite Nanoplatelets Composites using Two Processing Streams. Nigerian Journal of Environmental Sciences and Technology, 5(1), pp. 19-31. https://doi.org/10.36263/nijest.2021.01.0251 